\title{
Prevalence of different fungal species in Saliva and Swab samples of patients undergoing Radiotherapy for Oral Cancer
}

\author{
Pratima Putta $\mathbb{D}^{*}$, Kannan Natarajan $(\mathbb{D}$, Swapna Sridevi Beeraka (D), \\ Rakesh Kumar Manne (iD) and Prathi Venkata Sarath \\ Department of Oral Medicine and Radiology, Narayana Dental College, Nellore, Andhra Pradesh, India.
}

\begin{abstract}
Oral cancer is one of the leading causes of morbidity and mortality globally. There is a lack of studies on the growth of different Candida species in swab pre and post-radiotherapy. Hence, this study aimed to determine different Candida species in the oral cavity of patients who undergo radiotherapy. The study included 50 patients with oral cancer scheduled to undergo radiotherapy. Both saliva and swab samples were collected from all the patients before and after two- and six weeks of starting the radiotherapy. Similar inoculation was performed using the salivary swabs and incubated aerobically at $37^{\circ} \mathrm{C}$ for $48 \mathrm{~h}$. After $48 \mathrm{~h}$, culture plates were removed from the incubator and observed for the growth of different fungal species based on the color of the colonies. The culture of samples from patients with oral cancer resulted in the growth of $68 \%$ of Candida species in saliva and $44 \%$ in swab before initiating radiation. Unlike baseline findings, Candida albicans were identified in a higher number in swab samples (44\%) than saliva (24\%) post two weeks of radiotherapy. All saliva samples were positive to candida species, in which Candida albicans shows high prevalence (24\%). After six weeks of radiotherapy, in the swab, $30 \%$ of samples were positive for Candida albicans, and $18 \%$ of samples were positive for non-albicans species.Before radiotherapy, at two weeks and six weeks of radiotherapy, saliva samples showed a higher prevalence of Candidal species compared to swab samples. The combination of other Candida species was also higher in saliva samples compared to swab samples during radiotherapy.
\end{abstract}

Keywords: Candida albicans, oral cancer, saliva, swab, radiotherapy

*Correspondence: drprathima84@gmail.com

(Received: April 02, 2021; accepted: May 31, 2021)

Citation: Putta P, Natarajan K, Beeraka SS, Manne RK, Sarath PV. Prevalence of Different Fungal Species in Saliva and Swab Samples of Patients Undergoing Radiotherapy for Oral Cancer. J Pure App/ Microbiol. 2021;15(3):1180-1186. doi: 10.22207/JPAM.15.3.07

(C) The Author(s) 2021. Open Access. This article is distributed under the terms of the Creative Commons Attribution 4.0 International License which permits unrestricted use, sharing, distribution, and reproduction in any medium, provided you give appropriate credit to the original author(s) and the source, provide a link to the Creative Commons license, and indicate if changes were made. 


\section{INTRODUCTION}

Oral cancer comprises the malignancies within the oral cavity ${ }^{1}$. It ranks $6^{\text {th }}$ among the ten most common sites worldwide and $3^{\text {rd }}$ in the developing countries ${ }^{2}$. In India,Oral cancer is one of the leading causes of morbidity and mortality, partly because of malnutrition and habits of tobacco usage ${ }^{3,4}$.

Previous studies have shown an increase in the prevalence of Candida infections among oral squamous cell carcinoma patients receiving radiotherapy. Among different Candidal species, Candida abicans is identified as one of the common species isolated from the oral cavity of both healthy and medically impaired individuals $s^{5-7}$ and suggested that patients with head and neck undergoing radiotherapy must be prescribed for antifungal agents, along with topical fluoride, and the salivary substitutes. However, antifungal resistance trends have been transforming for Candida albicans and other Candida species. Hence, the necessity of isolating and defining the causative Candida species is gradually increasing in different populations ${ }^{8,9}$.

Radiotherapy may cause oral mucositis, ulceration, dysgeusia, and dysphagia. Oral ulcerations and the xerostomia have been reported to facilitate Candida growth ${ }^{4}$. Shifts within the bacterial flora may accompany these situations and allow Candida species to grow ${ }^{10}$.

Despite ionizing radiation as a popular treatment option for patients with head and neck cancer, Candida is the common genus that exists in human saliva. Due to their slow cell turnover rate, salivary glands should be relatively radio resistant, but improvements in salivary flow rate and consistency that arise immediately after radiotherapy imply that the gland tissue is an acutely responding tissue ${ }^{11}$. Compromise in immunologic mechanisms and oral clearance may lead to poor host protection, which is significantly linked with the changes in irradiated patients' oral microbiota. The most scientifically important variations in the oral microbiota caused by radiation are increases in Streptococcus mutans, Lactobacillus species, and the Candida species ${ }^{12,13}$. Candidasis, on the other hand, is the most prevalent microbial infection of oral cavity after radiotherapy. According to Ramirez-Amador et al. study, the prevalence of positive Candida cultures ranged from $43 \%$ at baseline to $62 \%$ after radiotherapy completion, and $75 \%$ during the follow-up ${ }^{14}$.

However, it is not clear whether the different treatment modalities affect the salivary Candida status of oral squamous cell carcinoma patients. There is lack of studies on comparative analysis of growth of different Candida species in swab pre and post-radiotherapy. Hence, current study designed to identify and compare various Candida species before and during the radiotherapy in both saliva and swab samples of the oral cavity of patients with oral cancer.

\section{MATERIALS AND METHODS}

This prospective non-randomized study with oral cancer who were scheduled to undergo radiotherapy at the Indian Red Cross Society, Cancer Hospital, Podalakuru Road, Nellore. Participants in the study were given detailed information about the study's intent and nature and the requirement to perform therapeutic examinations of the oral cavity at various intervals during radiotherapy before participating in the study. Patients who have been on antifungal medications, already had onco surgery and/or chemotherapy for oral cancer, are unwilling to participate in the study, or taking medicines that change immune response were excluded. Written informed consent of willingness and voluntary nature of participation in the study was taken from the participants. This study protocol was approved by Institutional ethics committee, Narayana Dental College and Hospital, Nellore, Andhra Pradesh.

\section{Sample collection}

This study comprises 50 patients with stage 3 and stage 4 oral cancer present in buccal mucosa, alveolar mucosa and tongue who scheduled for radiotherapy. All the included patients were examined clinically after a brief history of tobacco, areca nut chewing, smoking and alcohol consumption habits in terms of type, quantity, and duration. Prior sample collection, patients were allowed to sit in a quiet room and instructed not to drink, smoke, eat or clean their mouth $60 \mathrm{~min}$ before sample collection. Approximately $5 \mathrm{ml}$ of unstimulated saliva was collected from each patient in a sterile container by spitting method. Further, one more sample was collected by a sterile cotton swab from the lower 
labial sulcus by swabbing with single swipe from each patient. Both saliva and swab samples were collected from all the patients before and two and six-weeks of starting the radiotherapy.

Growth of fungal species using HICHROME Candida Differential Agar

Both saliva and swab samples were transported to the Laboratory of Microbiology at Narayana Medical College, Nellore. In order to minimize the effect of cell and food debris the collected saliva is treated by centrifugation at 3500 to $4000 \mathrm{rpm}$ before testing. The supernatant was separated from the sample, and sediment was inoculated on HICHROME Candida differential agar plates (HiMedia, Mumbai, India). Inoculation was performed using the swabs and incubated aerobically at $37^{\circ} \mathrm{C}$ for $48 \mathrm{~h}$. After $48 \mathrm{~h}$, culture plates were removed from the incubator and observed for the characteristic pigmented colonies with 50 to $100 \mathrm{CFU} / \mathrm{ml}$ growth of different fungal species was identified based on the color of the colonies.

1. C.albicans - Is seen in Light green colored colonies,

2. C.tropicalis - Seen as blue colonies.

3. C.glabrata - Seen as pink colonies,

4. C.krusei- Seen as purple colonies,

5. C. parapsilosis -Seen in cream-colored colonies,

6. C.dubliniensis- As Dark green colonies.

Statistical Analysis

The data obtained were tabulated and subjected to statistical analysis using IBM SPSS version 22 to evaluate the significant differences between different groups. Pearson Chi-square test was analyzed to determine the association between different fungal species in saliva and swab sample.

\section{RESULTS}

After growth in culture, fungi are identified based on visual characteristics such as colony morphology and color. The growth in differential agar is not only a definitive identification of the species. DNA sequence information has been used to identify fungal species. Matrix-assisted laser desorption ionization time-of-flight (MALDI-TOF) mass spectrometry holds considerable promise for identification of fungal species.

The current study conducted to identify various fungal species in saliva and swab samples of oral cancer patients before, after two weeks and six weeks of radiotherapy. Of 50 patients, 29 were men, and 21 were women with a median age of 51.72 years and 57.71 years.

Prevalence of different fungal species before radiotherapy

The culture of samples from oral cancer

Table 1. Percentages of salivary and swab samples of oral cancer patients showing growth of different fungal species before initiating radiotherapy

\begin{tabular}{lcc}
\hline Fungal Species & \multicolumn{2}{c}{ Before Radiation } \\
& $\begin{array}{c}\text { Saliva } \\
\text { (in \%) }\end{array}$ & $\begin{array}{c}\text { Swab } \\
\text { (in \%) }\end{array}$ \\
\hline C. albicans & 52 & 40 \\
C. albicans + C. krusei & 2 & \\
C. parapsilosis + C. glabrata & 6 & \\
C. albicans + C. tropicalis & 8 & 2 \\
C.albicans + C.parapsilosis & & 2 \\
No Candidal species & 32 & 56 \\
\hline
\end{tabular}
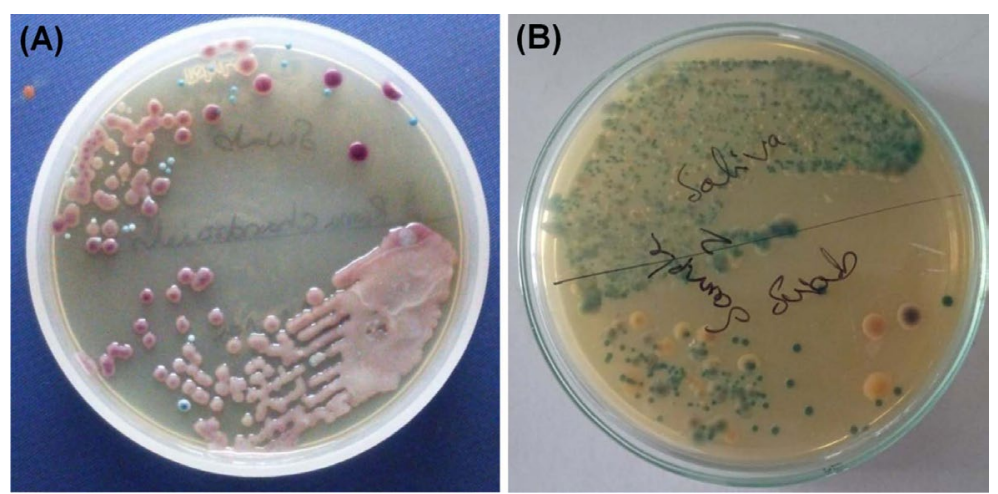

Fig. 1. Growth of different fungal colonies from salivary and swab samples (A) Candida albicans (B) Candida tropicalis 
patients resulted in the growth of Candida species was $68 \%$ in saliva and $44 \%$ in swab before initiating radiation which was statistically significant $(p=0.0001)$. The highest prevalence was observed for Candida albicans in both saliva $(n=26,52 \%)$ and swab ( $n=20,40 \%)$. Comparatively higher growth of non-albican species observed were $2 \%$ Candida krusei, 6\% Candida glabrata, and 8\% Candida tropicalis along with Candida albicans in saliva and $2 \%$ Candida tropicalis, 2\% Candida parapsilosis along with Candida albicans in swab samples. $32 \%$ of saliva samples and $56 \%$ of swab samples did not show any Candida species' growth (Table 1).

Prevalence of different fungal species after two weeks of radiotherapy

The prevalence of different Candidal species in salivary samples after two weeks of radiotherapy were highest in Candida albicans (24\%) followed by Candida albicans + Candida parapsilosis (14\%), Candida albicans + Candida glabrata (12\%), Candida albicans + Candida tropicalis (12\%). C.albicans + C. tropicalis $+C$. dubliniensis (6\%), C.albicans + C.dubliniensis (6\%), C.parapsilosis + C.glabrata (4\%), and C.parapsilosis + C. tropicalis (4\%). The least
Candidal species observed were C. Ibicans + C.krusei (2\%), C. tropicalis +C. parapsilosis (2\%). Swab samples showed the highest prevalence of Candida albicans (44\%) followed by Candida albicans + Candida tropicalis (14\%), C.albicans + C.parapsilosis (8\%), C.parapsilosis (4\%), C.albicans + C.glabrata (4\%), C.tropicalis (2\%), C.albicans + C.parapsilosis + C.glabrata (2\%), C.albicans + C.tropicalis + C.glabrata (2\%). $20 \%$ of swab samples did not show growth of Candida species. This increase in non albicans species in saliva and swab samples has shown a highly statistically significance $(p<0.0001)$ (Table 2$)$.

Prevalence of different fungal species after six weeks of radiotherapy

Salivary samples after six weeks of radiotherapy showed Candida albicans (26\%) followed by Candida albicans + Candida glabrata and Candida albicans + Candida tropicalis (10\% each), and Candida albicans + Candida parapsilosis (6\%). The least seen species were Candida dubliniensis (2\%), Candida albicans + Candida krusei (2\%), and combinations of Candida albicans with Candida dubliniensis and Candida glabrata and Candida parapsilosis (2\%). No candidal species

Table 2. Percentages of salivary and swab samples of oral cancer patients showing growth of fungal species after two- and six-weeks of radiotherapy

\begin{tabular}{|c|c|c|c|c|}
\hline \multirow[t]{2}{*}{ Fungal Species } & \multicolumn{2}{|c|}{2 Weeks } & \multicolumn{2}{|c|}{6 Weeks } \\
\hline & $\begin{array}{l}\text { Saliva } \\
\text { (in \%) }\end{array}$ & $\begin{array}{l}\text { Swab } \\
\text { (in \%) }\end{array}$ & $\begin{array}{l}\text { Saliva } \\
\text { (in \%) }\end{array}$ & $\begin{array}{l}\text { Swab } \\
\text { (in \%) }\end{array}$ \\
\hline C. albicans & 24 & 44 & 26 & 30 \\
\hline C.glabarata & & & & 4 \\
\hline C.dubliniensis & & & 2 & \\
\hline C.parapsilosis & & 4 & 4 & 4 \\
\hline C.tropicalis & & 2 & & \\
\hline C.albcansi+ C.dubliniensis & 6 & & 4 & \\
\hline C.albicans+C.glabrata & 12 & 4 & 10 & \\
\hline C.albicans+C.krusei & 2 & & 2 & \\
\hline C.albicans+C.parapsilosis & 14 & 8 & 6 & 2 \\
\hline C.parapsilosis+ C.glabarata & 4 & & 4 & \\
\hline C.parapsilosis + C.tropicalis & 6 & & 4 & 2 \\
\hline C.albicans+ C.tropicalis & 12 & 14 & 10 & 4 \\
\hline C.albicans + C. parapsilosis + C.glabrata & 6 & 2 & 2 & \\
\hline C.albicans+C.parapsilosis+C.krusei & 2 & & & \\
\hline C.albicans+C.tropicalis+C.dubliniensis & 6 & & 4 & \\
\hline C.albicans+C.tropicalis+C.glabrata & 6 & 2 & 6 & 2 \\
\hline C.albicans+C.dubliniensis+C.glabarata & & & 2 & \\
\hline No Candidal growth & & 20 & 14 & 52 \\
\hline
\end{tabular}


growth seen in $14 \%$ of saliva sample (Table 2 ).

Swab after 6 weeks of radiotherapy showed the highest prevalence of Candida albicans (30\%) followed by Candida albicans + Candida tropicalis, Candida glabrata, and Candida parapsilosis ( $4 \%$ each). $52 \%$ of samples did not show growth of Candida species in swab. The statistical analysis between saliva and swab samples after six weeks of radiotherapy showed non-significance $(p<0.229)$ (Table 2).

\section{DISCUSSION}

Candidiasis is caused by a yeast-like fungus called Candida. The rising prevalence of head and neck cancer is a serious public health concern, and radiotherapy plays a critical role in the treatment of such cases. Higher prevalence of Candida in the oral cavity of patients on anticancer therapy compared with the normal individuals is due to the immune compromised state during radiotherapy. It is becoming increasingly important to improve the quality of life of treated patients. The existence of fungal species and their differences were investigated in 50 patients with oral cancer before radiotherapy and at different intervals after radiotherapy. As saliva and swab are effective and non-invasive diagnostic aids both salivary and swab samples were analyzed before beginning radiotherapy, two weeks, and six weeks after starting radiotherapy. HICHROM agar was used in this study to differentiate candidal species in the study population due to its sensitivity, simplicity, and speed of differentiation of different species of Candida yeasts.

Of the total enrolled patients with oral cancer, before radiotherapy saliva samples showed higher prevalence of Candidal species (68\%) compared to swab samples (44\%). Larger number of patients showed the presence of Candida albicans in saliva (52\%) compared to swab (40\%). The combination of other Candida species was also higher in saliva than swab $(p=0.0001)$. The prevalence of Candia and noncandidal species in saliva and swab samples were almost similar in both men and women. Earlier studies have demonstrated almost similar findings in comparatively lower number of patients ${ }^{7,15}$. The higher number of non-albicans species in the present study may be attributed to the ecology of pathogens and the evolution of non-albicans species as pathogens among immune-compromised individuals. These findings explored that saliva can be the better choice to identify different Candida species in patients with oral cancer to make better targeted choice of treatment. Because of increasing drug resistance among pathogenic fungi, the demand for antifungal susceptibility testing has increased, and for some fungal pathogens, isolation and speciation of the causative Candidal species by culture before beginning antifungal drug therapy may be advantageous for preventing the development of resistance to the drug.

In our study, unlike the baseline samples collected prior to initiate radiotherapy, after two weeks of radiotherapy, all the patients were positive for fungal growth for either albicans or non-albicans. Unlike baseline findings, Candida albicans were identified in higher number (almost two-fold) in swab samples (44\%) than saliva (24\%). All saliva samples gave positive indication for the growth of candida species representing highest for Candida albicans (24\%) followed with combinations of other species along with albicans. Overall in swab samples taken after two weeks of radiation, $38 \%$ of fungal species were non-albicans, $44 \%$ albicans, and $20 \%$ were negative for any fungal growth. These results were in accordance with other similar studies as the radiotherapy progresses, there is an increase in the incidence of non-albicans species ${ }^{16,17}$.

Overall in $60 \%$ of salivary samples, nonalbicans species were positive after six weeks of radiotherapy compared to $76 \%$ non-albicans after two weeks of radiotherapy. Whereas in swab samples taken 6 weeks after radiotherapy, 30\% samples were positive for Candida albicans, and the $18 \%$ samples were positive for non-albicans species. In $52 \%$ of patients, the swab samples were negative for any fungal elements. The statistical analysis has shown that a non-significant value $(p<0.229)$ indicates the probability of the decline in fungal species' trend with increasing radiation exposure duration. However, after six weeks of radiotherapy, the presence of non-albicans was observed in few patients (18\%). A recent study reported similar findings after two- and four-week radiotherapy; however, no statistically significant difference was observed ${ }^{7}$. Our findings are significantly different from others because our last 
screening period was much longer, i. e. six-weeks after radiotherapy. Because there was more time lag from prior radiation treatment, our patients had a reduction in the incidence of non-albicans species and a relatively lower incidence of albicans in swab samples, and about $52 \%$ of patients were negative for certain fungal species after six weeks of radiotherapy.

In summary, unlike most previous studies, two different types of samples (saliva and swab) were collected from each patient simultaneously, which increased the chances of comparative sensitivity for the detection of fungal organisms. Since this study is based on patients who only underwent radiotherapy as a therapeutic option, there are least confounding factors that could skew the findings.

\section{CONCLUSION}

The study revealed an increase incidence of Candidal species in both saliva and swab samples in the patients who are undergoing radiotherapy for oral cancer. At two weeks of radiotherapy there was increase in the growth of non-albican species like C.glabrata, C.tropicalis, C.parapsilosis and decrease in Candida albicans in both saliva and swab samples. There was an overall decrease in the prevalence of Candidal species in swab samples compared to salivary samples at six weeks of radiotherapy. Before radiotherapy, at two weeks and six weeks of radiotherapy saliva samples showed higher prevalence of Candidal species compared to swab samples. The combination of other Candida species was also higher in saliva samples compared to swab samples. However, the occurrence of Candida albicans was significantly decreased in the salivary and swab samples. Both the saliva and swab samples could be used for Hichrome agar culture to differentiate Candidial species in patients receiving radiotherapy for oral cancers to opt for effective therapeutic options by isolating and defining the causative Candidal species for preventing the species from developing resistance to the drug.

\section{ACKNOWLEDGMENTS}

None.

\section{CONFLICT OF INTEREST}

The authors declare that there is no conflict of interest.

\section{AUTHORS' CONTRIBUTION}

All authors listed have made a substantial, direct, and intellectual contribution to the work and approved it for publication.

\section{FUNDING}

None.

\section{DATA AVAILABILITY}

All datasets generated or analyzed during this study are included in the manuscript.

\section{ETHICS STATEMENT}

The study was approved by the Institutional Ethical Committee of Narayana Dental College and Hospital, Nellore-524002, A.P, India and was carried out in accordance with the approved guidelines.

\section{REFERENCES}

1. Jemal A, Siegel R, Xu J, Ward E. Cancer statistics. CA Cancer J Clin. 2010;60(5):277-300. doi: 10.3322/ caac. 20073.

2. Johnson N. Tobacco use and oral cancer: a global perspective. J. Dent. Educ. 2001;65(4):328-39. doi: 10.1002/j.0022-0337.2001.65.4.tb03403.x

3. Panghal M, Kaushal V, Kadayan S, Yadav JP. Incidence and risk factors for infection in oral cancer patients undergoing different treatments protocols. BMC Oral Health. 2012;12:22. doi: 10.1186/1472-6831-12-22

4. Rajendran R. Benign and Malignant tumors of Oral Cavity. In: Rajendran R, Sivapathasundaram B, Editors. Shafer's Textbook of Oral Pathology. $7^{\text {th }}$ Ed., Chapter 2, India: Elsevier. 2011;102.

5. Makinen Al, Makitie A, Meurman JH. Candida prevalence in saliva before and after oral cancer treatment. The Surgeon. 2021. doi: 10.1016/j. surge.2021.01.006

6. Lam-Ubol A, Matangkasombut O, Trachootham D, et al. Efficacy of gel-based artificial saliva on Candida colonization and saliva properties in xerostomic post-radiotherapy head and neck cancer patients: a randomized controlled trial. Clin Oral Investig. 2021;25(4):1815-1827. doi: 10.1007/s00784-02003484-1

7. Anjali $K$, Arun AB, Bastian TS, Parthiban $R$, Selvamani $M$, Adarsh $\mathrm{H}$. Oral microbial profile in oral cancer patients before and after radiation therapy in a cancer care center - A prospective study. J Oral Maxillofac Pathol. 2020;24(1):117-124. doi: 10.4103/jomfp. JOMFP_213_19 
8. Mc Quillen DP, Zingman BS, Meunier F, Levi SM. Invasive infections due to Candida krusei: Report of 10 cases of fungemia that include three cases of endophthalmitis. Clin Infect Dis. 1992;14(2):472-478. doi: 10.1093/clinids/14.2.472

9. Fidel PL Jr, Vazquez JA, Sobel JD. Candida glabrata: Review of epidmiology, pathogenesis and clinical disease with comparison to C. albicans. Clin Microbiol Rev. 1999;12(1):80-96. doi: 10.1128/CMR.12.1.80

10. Ellepola AN, Samaranayake LP. The postantifungal effect (PAFE) of antimycotics on oral C. albicans isolates and its impact on candidal adhesion. Oral Dis. 1998;4(4):260-267. doi: 10.1111/j.1601-0825.1998. tb00290.x

11. Doppalapudi R, Vundavalli S, Prabhat MP. Effect of probiotic bacteria on oral Candida in head- and neckradiotherapy patients: A randomized clinical trial. J Can Res Ther. 2020;16(3):470-477. doi: 10.4103/jcrt. JCRT_334_18

12. Cheng $\mathrm{SCH}$, Wu VWC, Kwong DLW, Ying MTC. Assessment of post-radiotherapy salivary glands. Br J Radiol. 2011;84(1001):393-402. doi: 10.1259/ bjr/66754762

13. Shrestha M, Boaz K, Srikant N, Shakya A. An assessment of candidal colonization and species differentiation in head and neck cancer patients receiving radiation. J Nepal Health Res Counc. 2014;12:156-161. doi: 10.33314/jnhrc.v0i0.546

14. Ramirez-Amador V, Silverman S Jr, Mayer P, Tyler M, Quivey J. Candidal colonization and oral candidiasis in patients undergoing oral and pharyngeal radiation therapy. Oral Surg Oral Med Oral Pathol Oral Radiol Endod. 1997;84(2):149-153. doi: 10.1016/S10792104(97)90061-5

15. de Freitas EM, Nobre SA, Pires MB, Faria RV, Batista AU, Bonan PR. Oral Candida species in head and neck cancer patients treated by radiotherapy. Auris Nasus Larynx. 2013;40(4):400-404. doi: 10.1016/j. anl.2012.11.011

16. Bakki SR, Kantheti LPC, Kuruba KK, Poosarla C, Baddam VRR, Mulakaluri RR. Candidal carriage, isolation and species variation in patients undergoing radiotherapy and chemotherapy for head and neck tumours. Journal of Dr. NTR University of Health Sciences. 2014;3(1):2834. doi: $10.4103 / 2277-8632.128427$

17. Jain $M$, Shah R, Chandolia B, et al. The Oral Carriage of Candida in Oral Cancer Patients of Indian Origin Undergoing Radiotherapy and/or Chemotherapy. J Clin Diagn Res. 2016;10(2):ZC17-ZC20. doi: 10.7860/ JCDR/2016/15702.7180. 